\title{
AIDS
}

\section{The relative contributions of HIV drug resistance, non-adherence and low-level viremia to viremic episodes on antiretroviral therapy in Sub-Saharan Africa \\ --Manuscript Draft--}

Manuscript Number:

Full Title:

Article Type:

Keywords:

Corresponding Author:

Corresponding Author Secondary

Information:

Corresponding Author's Institution:

Corresponding Author's Secondary

Institution:

First Author:

First Author Secondary Information:

Order of Authors:

Order of Authors Secondary Information:

Abstract:
AIDS-D-19-00963R1

The relative contributions of HIV drug resistance, non-adherence and low-level viremia to viremic episodes on antiretroviral therapy in Sub-Saharan Africa

Original paper (Epidemiology / Social)

Antiretroviral therapy, adherence, drug resistance, low-level viremia, virological failure, virological rebound, virological re-suppression, sub-Saharan Africa

Seth Chekata Inzaule

Amsterdam Institute for Global Health and Development

Amsterdam, NETHERLANDS

Amsterdam Institute for Global Health and Development

Seth Chekata Inzaule

Seth Chekata Inzaule

Silvia Bertagnolio

Cissy M. Kityo

Margaret Siwale

Sulaimon Akanmu

Maureen Wellington

Marleen de Jager

Prudence Ive

Kishor Mandaliya

Wendy Stevens

T. Sonia Boender

Pascale Ondoa

Kim C.E. Sigaloff

Tobias F. Rinke de Wit

Raph L. Hamers

Introduction: To achieve viral suppression among $>90 \%$ of people on antiretroviral therapy (ART), improved understanding is warranted of the modifiable causes of HIV viremic episodes (VEs). We assessed the relative contributions of drug-resistance, non-adherence and low-level viremia $(\mathrm{LLV})(\mathrm{VL}<1,000 \mathrm{cps} / \mathrm{mL})$ on VEs in sub-Saharan Africa.

Methods: In a multi-country adult cohort initiating non-nucleoside reversetranscriptase inhibitor(NNRTI)-based first-line ART, VEs (VL $\geq 1,000 \mathrm{cps} / \mathrm{mL})$ were classified as i)viral non-suppression at 12 months; ii)virological rebound at 24 months (after initial viral suppression at 12 months; iii)failure to achieve viral re-suppression at 
24 months (after VE at 12 months). We used adjusted odds ratios from multivariable logistic regression to estimate attributable fractions for each risk factor.

Results: Of 2,737 cohort participants, 1,935 had data on pre-treatment drugresistance (PDR) and at least $1 \mathrm{VL}$ outcome. Viral non-suppression episodes

$(173 / 1,935$ [8.9\%]) were attributable to non-adherence in $30 \%$ (35\% in men vs $24 \%$ in women) and to PDR to NNRTIs in $10 \%$ (15\% in women vs $6 \%$ in men). Notably, at contemporary PDR prevalences of $10-25 \%$, PDR would explain $13-30 \%$ of viral nonsuppression. Virological rebound episodes $(96 / 1,515$ [6.3\%]) were mostly attributable to LLV $(29 \%)$ and non-adherence $(14 \%)$, and only rarely to PDR $(1.1 \%)$. Failures to achieve viral re-suppression $(66 / 81$ [81.5\%]) were mostly attributable to the presence of acquired drug resistance (34\%) and only rarely to non-adherence $(2.4 \%)$.

Conclusions: Effective adherence interventions could substantially reduce viral nonsuppression (especially in men) and virological rebound (especially during LLV), but would have limited effect on improving viral re-suppression. Alternative ART regimens could circumvent PDR and acquired resistance. 


\section{Abstract}

Introduction: To achieve viral suppression among $>90 \%$ of people on antiretroviral therapy (ART), improved understanding is warranted of the modifiable causes of HIV viremic episodes (VEs). We assessed the relative contributions of drug-resistance, non-adherence and low-level viremia (LLV) (VL<1,000 cps/mL) on VE's in sub-Saharan Africa.

Methods: In a multi-country adult cohort initiating non-nucleoside reverse-transcriptase inhibitor(NNRTI)-based first-line ART, VEs (VL $\geq 1,000 \mathrm{cps} / \mathrm{mL}$ ) were classified as i)viral nonsuppression at 12 months; ii)virological rebound at 24 months (after initial viral suppression at 12 months; iii)failure to achieve viral re-suppression at 24 months (after VE at 12 months). We used adjusted odds ratios from multivariable logistic regression to estimate attributable fractions for each risk factor.

Results: Of 2,737 cohort participants, 1,935 had data on pre-treatment drug-resistance (PDR) and at least $1 \mathrm{VL}$ outcome. Viral non-suppression episodes (173/1,935 [8.9\%]) were attributable to non-adherence in $30 \%$ (35\% in men vs $24 \%$ in women) and to PDR to NNRTIs in $10 \%$ (15\% in women vs $6 \%$ in men). Notably, at contemporary PDR prevalences of $10-25 \%$, PDR would explain $13-30 \%$ of viral non-suppression. Virological rebound episodes (96/1,515 [6.3\%]) were mostly attributable to LLV (29\%) and non-adherence $(14 \%)$, and only rarely to PDR (1.1\%). Failures to achieve viral re-suppression (66/81 [81.5\%]) were mostly attributable to the presence of acquired drug resistance (34\%) and only rarely to non-adherence $(2.4 \%)$.

Conclusions: Effective adherence interventions could substantially reduce viral nonsuppression (especially in men) and virological rebound (especially during LLV) but would have limited effect on improving viral re-suppression. Alternative ART regimens could circumvent PDR and acquired resistance. 
The relative contributions of HIV drug resistance, non-adherence and low-level viremia to viremic episodes on antiretroviral therapy in Sub-Saharan Africa

\author{
Authors \\ Seth C. Inzaule1, Silvia Bertagnolio², Cissy M. Kityo ${ }^{3}$, Margaret Siwale ${ }^{4}$, Sulaimon Akanmu ${ }^{5}$, \\ Maureen Wellington ${ }^{6}$, Marleen de Jager ${ }^{7}$, Prudence Ive ${ }^{8}$, Kishor Mandaliya ${ }^{9}$, Wendy Stevens ${ }^{10}$, \\ T. Sonia Boender ${ }^{1}$, Pascale Ondoa ${ }^{1,11}$, Kim C.E. Sigaloff ${ }^{1,12,}$ Tobias F. Rinke de Wit ${ }^{1}$, Raph L. \\ Hamers $^{1,13 x}$
}

\title{
Author affiliations
}

1. Amsterdam Institute for Global Health and Development, and Department of Global Health, Amsterdam UMC, University of Amsterdam, Amsterdam, The Netherlands;

2. Department of HIVIAIDS, World Health Organization, Geneva, Switzerland

3. Joint Clinical Research Centre, Kampala, Uganda;

4. Lusaka Trust Hospital, Lusaka, Zambia;

5. Department of Haematology and Blood Transfusion, Lagos University Teaching Hospital, and College of Medicine of the University of Lagos, Lagos, Nigeria;

6. Newlands Clinics, Harare, Zimbabwe;

7. Muelmed Hospital, Pretoria, South Africa;

8. Clinical HIV Research Unit, University of the Witwatersrand, Johannesburg, South Africa

9. Coast Province General Hospital, Mombasa, Kenya

10. Department of Molecular Medicine and Haematology, University of the Witwatersrand, Johannesburg and the National Health Laboratory Service, Johannesburg, South Africa

11. African Society of Laboratory Medicine, Addis Ababa, Ethiopia.

12. Department of Internal Medicine, Division of Infectious Diseases, Amsterdam UMC, Amsterdam, The Netherlands

13. Eijkman-Oxford Clinical Research Unit, Jakarta, Indonesia, and Centre for Tropical Medicine and Global Health, Nuffield Dept of Medicine, University of Oxford, Oxford, UK;

\section{Corresponding author:}

Seth Inzaule; Email: s.inzaule@aighd.org

AHTC, Tower C4, Paasheuvelweg 25, 1105 BP Amsterdam, The Netherlands

Phone: +31202103960 


\section{Alternative corresponding author:}

Raph Hamers; Email: r.hamers@pharmaccess.org

AHTC, Tower C4, Paasheuvelweg 25, 1105 BP Amsterdam, The Netherlands

Phone: +31202103960

\section{Abstract}

Introduction: To achieve viral suppression among $>90 \%$ of people on antiretroviral therapy (ART), improved understanding is warranted of the modifiable causes of HIV viremic episodes (VEs). We assessed the relative contributions of drug-resistance, non-adherence and low-level viremia (LLV) (VL<1,000 cps/mL) on VEs in sub-Saharan Africa.

Methods: In a multi-country adult cohort initiating non-nucleoside reverse-transcriptase inhibitor(NNRTI)-based first-line ART, VEs (VL $\geq 1,000 \mathrm{cps} / \mathrm{mL}$ ) were classified as i)viral nonsuppression at 12 months; ii)virological rebound at 24 months (after initial viral suppression at 12 months; iii)failure to achieve viral re-suppression at 24 months (after VE at 12 months). We used adjusted odds ratios from multivariable logistic regression to estimate attributable fractions for each risk factor.

Results: Of 2,737 cohort participants, 1,935 had data on pre-treatment drug-resistance (PDR) and at least $1 \mathrm{VL}$ outcome. Viral non-suppression episodes (173/1,935 [8.9\%]) were attributable to non-adherence in $30 \%$ ( $35 \%$ in men vs $24 \%$ in women) and to PDR to NNRTIs in $10 \%$ ( $15 \%$ in women vs $6 \%$ in men). Notably, at contemporary PDR prevalences of $10-25 \%$, PDR would explain $13-30 \%$ of viral non-suppression. Virological rebound episodes (96/1,515 [6.3\%]) were mostly attributable to LLV (29\%) and non-adherence (14\%), and only rarely to PDR $(1.1 \%)$. Failures to achieve viral re-suppression (66/81 [81.5\%]) were mostly attributable to the presence of acquired drug resistance (34\%) and only rarely to non-adherence $(2.4 \%)$.

Conclusions: Effective adherence interventions could substantially reduce viral nonsuppression (especially in men) and virological rebound (especially during LLV), but would have limited effect on improving viral re-suppression. Alternative ART regimens could circumvent PDR and acquired resistance.

\section{Keywords}

Antiretroviral therapy, adherence, drug resistance, low-level viremia, virological failure, virological rebound, virological re-suppression, sub-Saharan Africa 


\section{Introduction}

The 2020 global targets of having $90 \%$ of all HIV infected people being diagnosed, $90 \%$ of those diagnosed being on antiretroviral treatment (ART), and $90 \%$ of patients on ART achieving viral suppression, mark the roadmap to elimination of the AIDS epidemic as a public health threat by 2030[1]. By December 2018, an estimated 62\% of people living with HIV in low and middleincome countries (LIMC) were receiving ART, of whom $86 \%$ had viral suppression - falling short of the 90 goals [2].

There are few data on the relative contributions of some of the factors associated with and predictive of viremic episodes (VEs) during ART in LMIC [3-5](Figure 1). Non-adherence to ART has been documented as a major determinant of VEs [6-8]. Drug resistance to the widely used non-nucleoside reverse transcriptase inhibitors (NNRTI) efavirenz and nevirapine, when present before ART initiation, has also been identified as a major determinant of failure to achieve initial viral suppression, and a driver of treatment switches [9-11]. The impact of pretreatment drug resistance (PDR) is particularly concerning as rising PDR prevalences between 10 to $25 \%$ have been reported across sub-Saharan Africa [12]. Drug resistance is also acquired during virological failure, especially due to suboptimal adherence, and is considered a key determinant for failure to achieve viral re-suppression despite interventions to improve adherence $[3,13,14]$. Low-level viremia (LLV), defined as a plasma viral load (VL) between 50$1000 \mathrm{cps} / \mathrm{ml}$, has been associated with virological rebound after initial viral suppression $[5,15,16]$ (Figure 1).

To inform intervention strategies to enhance sustained viral suppression, we aimed to untangle the relative contributions of the key modifiable risk factors, i.e. PDR and acquired drug resistance (ADR), non-adherence, and LLV, that are associated with VEs in ART-treated populations in sub-Saharan Africa. 


\section{Methods}

\section{Study design and population}

Pan-African Studies to Evaluate Resistance-Monitoring (PASER-M) was a prospective cohort of HIV-1-infected adults ( $\geq 18$ years) conducted between 2007 and 2014, at 13 sites in six African countries (Kenya, Nigeria, South Africa, Uganda, Zambia, Zimbabwe), as profiled previously [9]. Care and treatment was based on local standard of care. For the present analysis, we included all participants who received first-line ART containing an NNRTI plus two NRTIs, who had a genotypic resistance test result available at ART initiation and a VL test result at year 1 and/or 2. Self-reported 30-day adherence was recorded every 3 months using visual analogue score (VAS), with a participant classified as non-adherent if the 12- or 24-months average VAS was $\leq 95 \%$. Demographic, clinical and laboratory data were collected using standard case report forms which were aggregated in a Web-based data system. All participants provided written informed consent. The study was approved by the national and local research ethics committees at the collaborating sites and by the Amsterdam UMC, University of Amsterdam (Amsterdam, Netherlands).

\section{Virological analysis}

HIV VL was determined on cryopreserved plasma samples at ART initiation and annually thereafter, using NucliSens EasyQ real-time (version 2.0; bioMérieux, Lyon, France) or COBAS Ampliprep/COBAS Taqman assay (Roche, Branchburg, New Jersey). Sanger sequencing was done on samples with $V L \geq 1,000 \mathrm{cps} / \mathrm{ml}$; drug resistance (DR) was defined as the presence of $\geq 1$ major drug resistance mutation included in the International Antiviral Society-USA mutation list of December 2019 associated with the NNRTIs nevirapine or efavirenz [17]. VL measurements at month 12 and 24 of ART were classified as VE $(\geq 1,000 \mathrm{cps} / \mathrm{mL})$ or viral suppression ( $<1,000 \mathrm{cps} / \mathrm{mL}$ ) (WHO-defined threshold)[18], and additionally as LLV (50-999 $\mathrm{cps} / \mathrm{mL})$.

\section{Statistical analysis}

Descriptive statistics were summarized by frequencies and percentages. Multivariable logistic regression with robust standard errors (to account for clustering of observations within sites) was used to assess associations between:

(i) PDR and non-adherence (independent variables) and viral non-suppression at month 12 or at 24 (dependent variable), in all participants; 
(ii) PDR, non-adherence and LLV at month 12 (independent variables) and virological rebound at month 24 (after initial viral suppression at month 12) (dependent variable);

(iii) Drug resistance at month 12 (ADR) and non-adherence (independent varibles) and failure to achieve viral re-suppression at month 24 (after VE at month 12) (dependent variable).

Models were adjusted from a set of potential confounders: age, sex, calendar year of treatment initiation, prior antiretroviral use, type of NNRTI and NRTI, pre-ART VL and CD4 cell count, WHO clinical stage and body mass index. A two-sided $p$-value of $\leq 0.05$ was considered significant. Due to significant interactions (at $\alpha=0.05$ level), we reported stratified analyses by sex for the association between PDR and viral non-suppression at 12 and 24 months; and by ART regimen (tenofovir+XTC+EFV vs non-tenofovir+XTC+EFV/NVP) for the association between LLV and virological rebound [19,20].

Using the adjusted associations from these models, we estimated attributable fractions (AFs) among the exposed and population attributable fractions (PAF). We estimated the proportions of VEs that were due to PDR, non-adherence, and LLV, respectively. The PAF was defined as the proportion of VEs that could have been prevented in case the risk factor was averted (i.e. optimal adherence ( $\geq 95 \%$ ), absence of resistance and absence of LLV). We estimated the PAFs based on maximum likelihood method[21] using the punaf syntax in Stata version 12 [22].

\section{Results}

\section{Patient characteristics}

Of 2,737 participants who initiated first-line ART, 1,935 (71\%) had data on PDR and a VL result after year 1 and/or 2 of ART. 60\% were women, median age was 37 (IQR 32-43) years. Initial ART regimens were TDF+XTC+EFV (33\%), non-TDF+XTC+EFV (30\%), non-TDF+XTC+NVP (37\%). The proportion of participants with PDR was 4.1\% (80/1941), and with non-adherence was $13.0 \%(252 / 1,935)$ in month $0-12,7.4 \%(112 / 1515)$ in month $12-24$, and $12.7 \%(221 / 1744)$ in month 0-24. PDR and non-adherence levels did not differ between sexes.

\section{Risk factors of viremic episodes}

\section{Viral non-suppression at month 12 and 24}


The proportion of participants with viral non-suppression was $8.9 \%(173 / 1,935)$ at month 12 with $10.9 \%(85 / 779)$ in men and $7.6 \%(88 / 1156)$ in women $(p=0.013)$, and $11 \%(192 / 1744)$ at month 24 -with 11\%, (117/1060) in women and 11\% (75/684) in men ( $p=0.962)$.

PDR was independently associated with the risk of viral non-suppression at month 12 (aOR 5.6, 95\% Cl 2.3-14.1) and month 24 (aOR 5.4, 95\% Cl 2.1-13.7), and this effect was about two times greater in women than men ( $p$ for interaction=0.013) (see Table 1). Non-adherence was associated with viral non-suppression at month 12 (aOR 6.0, 95\% Cl 4.4-8.1) and month 24 (aOR 5.3, 95\% $\mathrm{Cl} 4.0-7.0$ ), and this effect was greater in men than women at month 12, but not at month 24 (Table 1).

\section{Virological rebound}

$1,515(78.3 \%)$ of the participants had viral suppression at month 12 and a VL result available at month 24. The proportion of participants with virological rebound at month 24 was $6.3 \%$ (96/1,515).

PDR was not independently associated with the risk of virological rebound (aOR 1.4, 95\% Cl 0.46-4.1).

The proportion of participants with LLV at month 12 was $9.1 \%(138 / 1515)$, and LLV was independently associated with the risk of virological rebound (aOR 6.4, 95\% Cl 3.8-10.7), and this effect was greater among participants on non-TDF+XTC+NVP/EFV compared to those on TDF+XTC+EFV ( $p$ for interaction=0.025) (Table 2).

Non-adherence was independently associated with the risk of virological rebound (aOR 3.5, 95\% Cl 2.1-5.7) (Table 2).

\section{Failure to achieve viral re-suppression}

For 81 of 173 (47\%) participants who had VE at month 12, a genotypic resistance test result at month 12 and VL test result at month 24 was available. Overall 81.5\% (66/81) failed to achieve viral re-suppression at month 24 .

Among participants with VE at month 12, 69\% (56/81) had one or more acquired resistance mutations, and only 5.4\% (3/56) achieved viral re-suppression. Acquired drug resistance was strongly associated with failure to achieve viral re-suppression (aOR 18.1, 95\% Cl 3.5-93.7).

The proportion of participants who had non-adherence in month 12-24 was $21 \%$ (17/81), but this was not independently associated with failure to achieve viral re-suppression (aOR 2.3, 95\% Cl 0.89-6.0). 


\section{Attributable fractions for viremic episodes}

\section{Viral non-suppression at month 12 and 24}

Summaries of attributable fractions are shown in Table 3. In the cohort, the prevalence of PDR was $4.1 \%$, and the fraction of viral non-suppression attributed to PDR was $10.3 \%(95 \% \mathrm{Cl} 2.7 \%$ $17.3 \%)$ at month 12 and $7.1 \%(95 \% \mathrm{Cl} 1.9 \%-12.1 \%)$ at month 24 . This can be interpreted as, that $10.3 \%$ and $7.1 \%$ of the viral non-suppression episodes could have been averted by controlling for PDR. The fraction of viral non-suppression attributed to PDR were consistently higher in women compared to men (Table 1). When estimating the fractions of viral nonsuppression for populations with contemporary prevalence estimates of PDR $(10-25 \%$ as reported by $\mathrm{WHO}[12])$, the attributable fractions are estimated at 13-30\% (Supplementary figure 1).

In the overall cohort, the prevalence of non-adherence was $13 \%$ in month $0-12$ and $12.7 \%$ in month $0-24$, and the fraction of viral non-suppression episodes attributed to nonadherence was $29.5 \%(95 \% \mathrm{Cl}, 22.0 \%-36.2 \%)$ in month $0-12$, and $25.8 \%$ (95\% Cl, $19.7 \%$ $31.5 \%$ ) in month $0-24$; and was higher for men in month 0-12 only (Table 1). This can be interpreted as that ensuring optimal ART adherence could avert $30 \%$ of viral non-suppression episodes in the first year and $26 \%$ during the first two years of ART. Similarly, across a range of plausible prevalences of non-adherence at $10-30 \%$, the fractions of viral non-suppression attributable to non-adherence would be $15-37 \%$. (Supplementary figure 1).

\section{Virological rebound}

In the cohort, the fraction of virological rebound episodes attributable to PDR was $1.1 \%(95 \% \mathrm{Cl}$ $-3.1 \%-5.1 \%$ ) (Table 2). The fraction of virological rebound episodes attributable to nonadherence was $13.7 \%(95 \% \mathrm{Cl} 6.4 \%-20.3 \%)$ and did not significantly vary between ART regimens (Table 2). The fraction of virological rebound episodes attributable to LLV was $28.6 \%$ (95\% Cl, 18.0\%-37.9\%), and was higher in participants on non-TDF+XTC+EFV/NVP than those on TDF+XTC+EFV (Table 2). These findings can be interpreted as that in this cohort controlling for PDR, non-adherence or LLV could avert $1.4 \%, 13.7 \%$ and $28.6 \%$ of virological rebound episodes, respectively.

Across a range of plausible prevalences of PDR (10-25\%, non-adherence (10-30\%) and LLV (5-15\%), the attributable fractions of virological rebound espisodes was estimated to range between $3-6 \%, 10-28 \%$ and $8-23 \%$, respectively (Supplementary figure 2). 


\section{Failure to achieve viral re-suppression}

In the cohort, the fraction of failure to achieve viral re-suppression attributable to non-and to acquired DR was $33.9 \%(95 \% \mathrm{Cl} 21.5-55.4 \%)$. whereas the fraction of failure to achieve viral resuppression attributable to non-adherence was $2.4 \%(95 \% \mathrm{Cl}-0.39 \%-5.2 \%)$.

Across a range of plausible prevalences of acquired resistance (60-90\%) and nonadherence (10-30\%), attributable fractions of failures to achieve viral resuppression were higher for acquired resistance (37\%-42\%) than non-adherence (1-3\%) (Supplementary figure 3).

\section{Discussion}

This study provides novel insights into the relative contributions of the key potentially modifiable risk factors for viremic episodes in patients receiving first-line NNRTI-based ART in subSaharan Africa. Viral non-suppression episodes were attributable mainly to non-adherence but also to PDR. On the other hand, virological rebound episodes were mostly attributable to LLV and non-adherence and rarely to PDR. Failures to achieve viral re-suppression were mostly attributable to the presence of acquired drug and only rarely to non-adherence.

Overall non-adherence accounted for the largest fraction of cohort participants with viral nonsuppression (30\% in the first year, $26 \%$ in the first two years of ART). Addressing the challenges of adherence, especially early during ART, therefore remains a key intervention for improving the global target for viral suppression [23].

In this cohort, PDR accounted for a smaller, but nonetheless important fraction of viral non-suppression (10\% in the first year, $7 \%$ in the second year of ART). This finding is particularly relevant given that recent data suggest high prevalence of NNRTI-associated PDR in many settings in sub-Saharan Africa [12]. The recent WHO HIVDR report indicates that, of the 18 countries that monitored PDR between 2014 and 2018, 12 reported national estimates between $10-25 \%$ [12]. In those settings, PDR could potentially explain a substantial (13-30\%) fraction of viral non-suppression episodes. Moreover, our study adds that PDR explains a much higher fraction of viral non-suppression for women (15\%) than for men $(6 \%)$. These findings therefore provide further support to ongoing or planned efforts in many LMIC to accelerate the transition from NNRTI- to dolutegravir (DTG)-based first-line ART to circumvent the increasing impact of NNRTI-associated PDR [24]. 
By contrast, our findings suggested that PDR plays a negligible role in virological rebound, explaining only $\sim 1 \%$ of the episodes. These findings are in line with a recent study in South African women initiating ART during pregnancy which found that PDR plays a minor role in explaining virological rebound after initial viral suppression $(<10 \%)$, while non-adherence accounted for the vast majority (>90\%) [4].

We estimated that the largest fraction of virological rebound could be explained by the occurrence of LLV (29\%), in addition to non-adherence (14\%). It should be noted, however, that the attributable fraction of LLV was much larger for the less potent ART regimens, based on thymidine analogues and nevirapine, rather than TDF+XTC+EFV (Atripla) (32\% vs 6\%). This hypothesis is supported by earlier observations of higher potency of TDF+XTC+EFV and its availability as a fixed-dose combination $[20,25]$. Our study finding expands on previous studies, including a large study in South Africa, which found that patients with LLV are at higher risk of virological failure and decreased durability of viral re-suppression [5]. In addition, LLV has been correlated with increased risk of drug resistance, immunological failure, non-AIDS comorbidities, clinical progression to AIDS, death as well as potential increased risk of HIV transmission.[26] This suggest potential programmatic benefits of using lower viral load thresholds to trigger clinical actions such as adherence interventions.

Available data from the literature indicate that $60-90 \%$ of patients failing NNRTI-based regimen have acquired drug resistance mutations [14]. Among cohort participants with VE at month $12,69 \%$ had acquired at least one drug resistance mutation. Presence of acquired resistance accounted for a high attributable fraction (34\%) of patients who failed to achieve viral re-suppression on their first-line ART regimen at 24 months. A study from a routine clinical setting in Uganda that found that only $26 \%$ of patients with VE and detected resistance resuppressed on a continued first-line ART even with high-level adherence [3]. In our cohort, only $18.5 \%$ achieved viral re-suppression overall, and in those who had acquired resistance only $5.4 \%$ achieved re-suppression. This highlights the potential limited benefits of adherence interventions during late diagnosis of virological failure in patients failing on NNRTI-based treatment, warranting rapid switching to second-line therapy instead [3,27].

The main strengths of this study are its prospective multi-country design, and the setting of high-burden countries with increasing levels of PDR, which enhances its relevance for policy makers. Our study has some limitations. First, the study focused on NNRTI-based first-line ART only. Further studies are needed to provide insights on the etiology of VE with integrase 
inhibitors, following the transition to dolutegravir-based ART. Second, our study relied on a selfreported adherence measure, which, although valid and widely used, has potential for underestimating the effect of non-adherence on VE [28-30]. Viral load and therapeutic drug monitoring tests are more objective and are thus preferred alternatives. Moreover, we did not collect data on underlying determinants of non-adherence and thus could not be able to elucidate the observed sex-differences.

Third, there were few participants with the characteristic and outcome of interest in some of the models leading to wide Cls. This highlights the need for additional meta-analysis to precisely determine the attributable fractions of key determinants of VEs. Lastly, frequency of viral load testing was limited in our cohort and we did not collect information on enhanced adherence counselling among those with VE. Despite this, our data have relevance for current practice given that many LMIC still have substantial gaps in their viral load coverage and cascade.[12]

\section{Conclusion}

In conclusion, our study provides insight into the relative contribution of potential modifiable factors for VEs in people living with HIV who receive ART, that could be addressed to improve attainment of the UNAIDS-defined global target for $>90 \%$ viral suppression. Our findings suggest an important role of PDR to NNRTIs on failure to achieve initial viral suppression, but not in viral rebound. Viral rebound is predominantly attributable to non-adherence and LLV. High rates of acquired resistance limit the ability to achieve viral re-suppression among those failing treatment, prompting need for rapid regimen switching. Our study findings recommend exploring more sensitive VL thresholds to detect VE, enhanced adherence interventions particularly early during ART and accelerated rollout of dolutegravir first-line ART in populations with high PDR prevalence, to circumvent the negative impact of emerging NNRTI-associated PDR.

\section{Competing interests}

All authors declare that they have no conflict of interest.

\section{Acknowledgements}

The authors thank the study participants, the staff at the collaborating clinical sites and reference laboratories. PASER is part of the Linking African and Asian Societies for an Enhanced Response to HIVIAIDS (LAASER) program, a partnership of Stichting Aids Fonds, 
the Foundation for AIDS Research (amfAR)-TREAT Asia, PharmAccess Foundation, and International Civil Society Support (ICSS).

\section{Funding}

The Pan-African Studies to Evaluate Resistance (PASER) is an initiative of the Amsterdam Institute for Global Health and Development, with major support provided by the Ministry of Foreign Affairs of The Netherlands through a partnership with Stichting Aids Fonds (grant no. 12454) and The Netherlands Organization for Scientific Research (NWO-WOTRO grant no. W07.10.101 and W07.10.106), and additional support provided by De Grote Onderneming, The Embassy of the Kingdom of the Netherlands, Heineken Africa Foundation and Jura Foundation.

\section{Authors' contributions}

TFRW is the PASER principal investigator. CMK, MS, SA, MW, MDJ, PI, KM, WS, TSB, PO, KCES, TFRW and RLH established the cohort and supervised data collection. RLH and SCI conceived the study. SCl performed the statistical analysis, with input from RLH and SB. SCl, TFRW, SB and RLH drafted the manuscript. All authors provided valuable input to interpretation of the data and critically reviewed the paper for important intellectual content. All authors reviewed and approved the final version of the manuscript.

\section{References}

1. UNAIDS. Fast-Track - Ending the AIDS epidemic by 2030. 2014.

https://www.unaids.org/sites/default/files/media_asset/JC2686_WAD2014report_en.pdf (accessed March 09, 2020).

2. UNAIDS. Global AIDS update 2019 - Communities at the centre: Defending rights, breaking barriers, reaching people with HIV services. 2019.

https://www.unaids.org/sites/default/files/media_asset/2019-global-AIDS-update_en.pdf (accessed March 09, 2020).

3. McCluskey SM, Musinguzi N, Kamelian K, et al. HIV drug resistance as a significant driver of first-Line treatment failure in Uganda. Conference on Retroviruses and Opportunistic Infections (CROI). 2018.

4. Myer L, Redd AD, Mukonda E, et al. Antiretroviral adherence, elevated viral load and drug resistant mutations in HIV-infected women initiating treatment in pregnancy: a nested case-control study. Clin Infect Dis 2019; 
5. Hermans LE, Moorhouse M, Carmona S, et al. Effect of HIV-1 low-level viraemia during antiretroviral therapy on treatment outcomes in WHO-guided South African treatment programmes: a multicentre cohort study. Lancet Infect Dis 2017;

6. Mills EJ, Nachega JB, Bangsberg DR, et al. Adherence to HAART: a systematic review of developed and developing nation patient-reported barriers and facilitators. PLoS Med 2006; 3:e438.

7. Langebeek N, Gisolf EH, Reiss P, et al. Predictors and correlates of adherence to combination antiretroviral therapy (ART) for chronic HIV infection: a meta-analysis. BMC Med 2014; 12:142.

8. Bijker R, Jiamsakul A, Kityo C, et al. Adherence to antiretroviral therapy for HIV in subSaharan Africa and Asia: A comparative analysis of two regional cohorts. J Int AIDS Soc 2017;

9. Hamers RL, Schuurman R, Sigaloff KC, et al. Effect of pretreatment HIV-1 drug resistance on immunological, virological, and drug-resistance outcomes of first-line antiretroviral treatment in sub-Saharan Africa: a multicentre cohort study. Lancet Infect Dis $2012 ; 12: 307-317$.

10. Boender TS, Hoenderboom BM, Sigaloff KCE, et al. Pretreatment HIV drug resistance increases regimen switches in sub-Saharan Africa. Clin Infect Dis 2015; 61:1749-1758.

11. World Health Organization. Guidelines on the public health response to pretreatment HIV drug resistance. 2017. http://apps.who.int/iris/bitstream/10665/255880/1/9789241550055eng.pdf?ua=1 (accessed March 09, 2020).

12. World Health Organization. HIV drug resistance report:2019. https://www.who.int/hiv/pub/drugresistance/hivdr-report-2019/en/ (accessed March 09, 2020).

13. Hoffmann CJ, Charalambous S, Sim J, et al. Viremia, resuppression, and time to resistance in human immunodeficiency virus (HIV) subtype $\mathrm{C}$ during first-line antiretroviral therapy in South Africa. Clin Infect Dis 2009; 49:1928-1935.

14. Boender TS, Kityo CM, Boerma RS, et al. Accumulation of HIV-1 drug resistance after continued virological failure on first-line ART in adults and children in sub-Saharan Africa. J Antimicrob Chemother 2016; 71:2918-2927.

15. Geretti AM, Smith C, Haberl A, et al. Determinants of virological failure after successful viral load suppression in first-line highly active antiretroviral therapy. Antivir Ther 2008; 13:927-936.

16. Young J, Rickenbach M, Calmy A, et al. Transient detectable viremia and the risk of viral 
rebound in patients from the Swiss HIV Cohort Study. BMC Infect Dis 2015; 15:382.

17. Wensing AM, Vincent C, Ceccherini-Silberstein F, et al. 2019 Update of the Drug Resistance Mutations in HIV-1. 2019. https://www.iasusa.org/wpcontent/uploads/2019/07/2019-drug-resistance-mutations-figures.pdf (accessed March 09, 2020).

18. World Health Organization. Consolidated guidelines on the use of antiretroviral drugs for treating and preventing HIV infection Recommendations for a public health approach Second edition. 2016.

http://apps.who.int/iris/bitstream/handle/10665/208825/9789241549684_eng.pdf?sequen ce=1 (accessed March 09, 2020).

19. Kanters S, Vitoria M, Doherty M, et al. Comparative efficacy and safety of first-line antiretroviral therapy for the treatment of HIV infection: a systematic review and network meta-analysis. Lancet HIV 2016; 3:e510-e520.

20. Inzaule SC, Rinke de Wit TF, Hamers RL. Does Tenofovir-containing First-line Antiretroviral Therapy Mitigate the Impact of Pretreatment Non-nucleoside Reverse Transcriptase Inhibitor Drug Resistance? Clin Infect Dis 2019; 68:2158-2160.

21. Greenland S, Drescher K. Maximum Likelihood Estimation of the Attributable Fraction from Logistic Models. Biometrics 1993; 49:865-872.

22. Newson RB. Attributable and unattributable risks and fractions and other scenario comparisons. Stata J 2013;

23. Bonner K, Mezochow A, Roberts T, Ford N, Cohn J. Viral load monitoring as a tool to reinforce adherence: a systematic review. J Acquir Immune Defic Syndr 2013; 64:74-78.

24. World Health Organization. Update of recommendations on first- and second-line antiretroviral regimens Policy brief. Available at:

https://apps.who.int/iris/bitstream/handle/10665/325892/WHO-CDS-HIV-19.15eng.pdf?ua=1 (accessed March 09, 2020.

25. Derache A, Iwuji CC, Baisley K, et al. Impact of next generation sequencing defined HIV pre-treatment drug resistance on virological outcomes in the ANRS 12249 treatment as prevention trial. Clin Infect Dis 2018;

26. Ryscavage P, Kelly S, Li JZ, Richard Harrigan P, Taiwo B. Significance and clinical management of persistent low-level viremia and very-low-level viremia in HIV-1-infected patients. Antimicrob Agents Chemother 2014;

27. Pirkle CM, Boileau C, Nguyen V-K, et al. Impact of a modified directly administered antiretroviral treatment intervention on virological outcome in HIV-infected patients 
treated in Burkina Faso and Mali. HIV Med 2009; 10:152-156.

28. Aurpibul L, Teerananchai S, Prasitsuebsai W, et al. Therapeutic Drug Monitoring of Lopinavir in HIV-Infected Children on Second-Line Antiretroviral Therapy in Asia. Ther Drug Monit 2016; 38:791-795.

29. Inzaule SC, Hamers RL, Noguera-Julian M, et al. Clinically relevant thresholds for ultrasensitive HIV drug resistance testing: a multi-country nested case-control study. Lancet HIV 2018;

30. Alcaide ML, Ramlagan S, Rodriguez VJ, et al. Self-Report and Dry Blood Spot Measurement of Antiretroviral Medications as Markers of Adherence in Pregnant Women in Rural South Africa. AIDS Behav 2017; 21:2135-2140. 


\section{Figure legend}

Figure 1: A schematic illustration of viremic episodes during antiretroviral treatment in HIV infected patients

Supplementary figure 1: Estimated attributable fractions of viral non-suppression attributable to PDR and non-adherence, overall and by sex

Attributable fractions for viral non-suppression at 12 months and 95\% Cl (whiskers) associated with non-adherence (square boxes) and PDR (circles), expressed for all participants (white), women (black) and men (grey) against a range of plausible prevalences of either exposure ( $x$ axis).

Supplementary figure 2: Estimated attributable fractions of virological rebound attributable to PDR, non-adherence or low-level viremia, overall and by sex

Attributable fractions for virological rebound and 95\% Cl (whiskers) associated with nonadherence (square boxes), PDR (circles) and low-level viremia (rhombus), expressed for all patients (white), women (black) and men (grey) against a range of plausible prevalences of either exposure (x-axis).

Supplementary figure 3: Estimated attributable fraction of failure to achieve viral resuppression attributable to nonadherence and acquired drug resistance

Attributable fractions for viral re-suppression and 95\% Cl (whiskers) associated with nonadherence (square boxes) and acquired drug resistance (circles) against a range of plausible prevalences of either exposure (x-axis). 
Table 1: Viral non-suppression attributable to pretreatment drug resistance or non-adherence after 12 and 24 months of ART

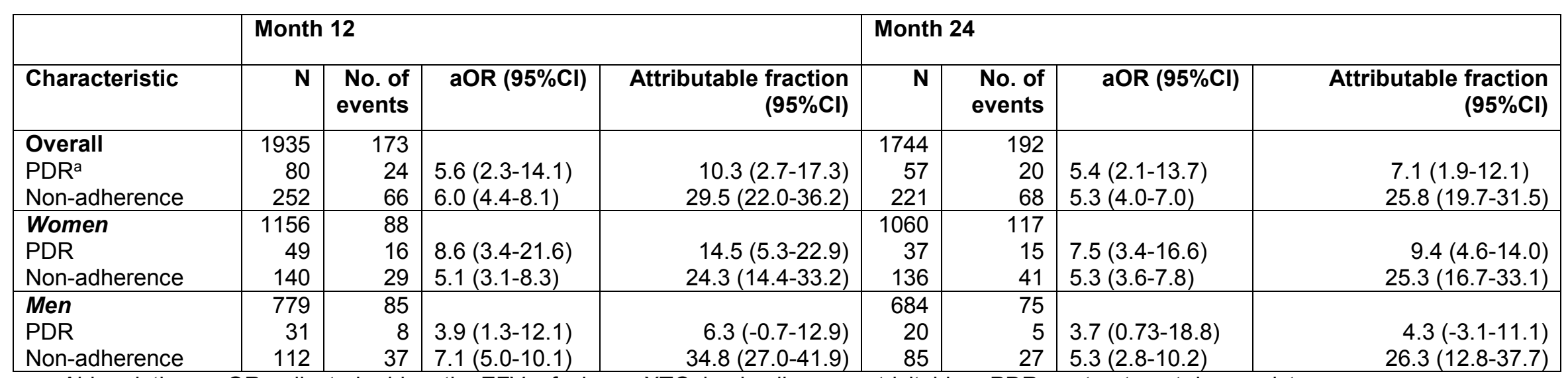

Abbreviations: aOR, adjusted odds ratio; EFV, efavirenz; XTC, lamivudine or emtricitabine; PDR, pretreatment drug resistance;

PDR was defined as any NNRTI mutation according to IAS mutation list 2019; Non-adherence was defined as mean VAS score <95\%.

Multivariable logistic regression analysis with odds ratios adjusted for age, sex, type of initial NNRTI and NRTI, WHO clinical stage, calendar year

of ART initiation, prior ARV exposure and pre-ART viral load and CD4 cell count.

a $p$ for interaction between PDR and sex was 0.017 at month 12 and 0.288 at month 24 
Table 2: Virological rebound attributable to pretreatment drug resistance, non-adherence or low-level viremia

\begin{tabular}{|l|r|r|l|r|}
\hline Characteristic & $\mathbf{N}$ & $\begin{array}{r}\text { No. of } \\
\text { events }\end{array}$ & aOR (95\%CI) & Attributable Fraction (95\% CI) \\
\hline Overall & 1515 & 96 & & \\
PDR & 41 & 5 & $1.4(0.46-4.1)$ & $1.1(-3.1-5.1)$ \\
Adherence & 112 & 22 & $3.5(2.1-5.7)$ & $13.7(6.4-20.3)$ \\
LLV at year 1 & 138 & 36 & $6.4(3.8-10.7)$ & $28.6(18.0-37.9)$ \\
\hline TDF+XTC+EFV & 408 & 16 & & \\
PDR & 13 & 0 & 1.0 & $12.4(-7.5-28.7)$ \\
Adherence & 24 & 3 & $3.5(0.77-16.3)$ & $5.5(-25.3-28.8)$ \\
LLV at year 1 & 34 & $1.5(0.22-10.6)$ & $2.0(-2.5-6.3)$ \\
\hline Non TDF+XTC+EFV/NVP & 1107 & 82 & & $14.3(6.6-21.4)$ \\
PDR & 28 & 5 & $1.7(0.58-5.2)$ & $32.2(23.7-39.7)$ \\
Adherence & 88 & 19 & $3.9(2.3-6.6)$ & \\
LLV at year 1 & 104 & 33 & $7.7(5.1-11.6)$ & \\
\hline
\end{tabular}

Abbreviations: aOR, adjusted odds ratio; EFV, efavirenz; XTC, lamivudine or emtricitabine; TDF, tenofovir; PDR, pretreatment drug resistance; $A D R$, acquired drug resistance; LLV, low level viremia

PDR and ADR defined as any NNRTI mutation according to IAS mutation list 2019; Adherence was defined as mean 30-day adherence with VAS score $<95 \%$ as non-adherence. LLV, defined as $50-1000 \mathrm{cps} / \mathrm{ml}$

Multivariable logistic regression analysis with odds ratios adjusted for; age, sex, type of initial NNRTI and NRTI, WHO clinical stage, BMI, calendar year of ART initiation, prior ARV exposure, pre-ART viral load and CD4 cell count.

a $p$ for interaction between LLV and type of ART regimen was 0.025 
Table 3: Relative contributions of various risk factors to viremic episodes among participants on first-line ART in sub-Saharan Africa

\begin{tabular}{|c|c|c|c|c|c|c|c|}
\hline & \multicolumn{3}{|c|}{$\begin{array}{c}\text { Viral non-suppression } \\
\text { (Month 12)* }\end{array}$} & \multicolumn{3}{|c|}{ Viral rebound (Month 24)* } & $\begin{array}{l}\text { Failure to achieve viral } \\
\text { re-suppression }\end{array}$ \\
\hline Potential modifiable risk factor & All & Men & Women & All & TDF/XTC/EFV & Non TDF/XTC/EFV & Attributable fraction \\
\hline $\begin{array}{l}\text { Pretreatment drug resistance } \\
\text { Cohort prevalence }(4.1 \%)^{\beta} \\
\text { Plausible prevalences }(10-25 \%)\end{array}$ & $\begin{array}{r}10 \% \\
10-30 \% \\
\end{array}$ & $\begin{array}{r}6 \% \\
10-24 \% \\
\end{array}$ & $\begin{array}{r}15 \% \\
17-36 \% \\
\end{array}$ & $\begin{array}{r}1 \% \\
3-6 \% \\
\end{array}$ & $\begin{array}{l}0 \\
0 \\
\end{array}$ & $\begin{array}{r}2.0 \% \\
4-11 \% \\
\end{array}$ & - \\
\hline $\begin{array}{l}\text { Non-adherence } \\
\text { Cohort prevalence } \beta \\
0-12 \text { months }(13 \%) \\
12-24 \text { months }(7.4 \%)^{\mathrm{a}} \\
12-24 \text { months }(21 \%)^{\mathrm{b}} \\
\text { Plausible prevalences }(10-30 \%)\end{array}$ & $\begin{array}{r}- \\
30 \% \\
- \\
- \\
-\end{array}$ & $\begin{array}{r}- \\
35 \% \\
- \\
- \\
\end{array}$ & $\begin{array}{r}- \\
24 \% \\
- \\
- \\
-\end{array}$ & $\begin{array}{r}- \\
- \\
14 \% \\
- \\
-\end{array}$ & $\begin{array}{r}- \\
- \\
12 \% \\
- \\
-\end{array}$ & $\begin{array}{r}- \\
- \\
14 \% \\
- \\
-\end{array}$ & $\begin{array}{r}- \\
2.4 \% \\
1-3 \% \\
\end{array}$ \\
\hline $\begin{array}{l}\text { Low-level viremia } \\
\text { Cohort prevalence }(9.1 \%) \\
\text { Plausible prevalences }(5-15 \%)\end{array}$ & $\begin{array}{l}- \\
-\end{array}$ & $\begin{array}{l}- \\
-\end{array}$ & $\begin{array}{l}- \\
-\end{array}$ & $\begin{array}{r}29 \% \\
8-23 \%\end{array}$ & $\begin{array}{r}6 \% \\
1.9-5.6 \%\end{array}$ & $\begin{array}{r}32 \% \\
9-24 \%\end{array}$ & - \\
\hline $\begin{array}{l}\text { Acquired drug resistance } \\
\text { Cohort prevalence }(69 \%)^{\beta} \\
\text { Plausible prevalences }(60-90 \%)\end{array}$ & - & - & - & - & $\begin{array}{l}- \\
-\end{array}$ & $\begin{array}{l}- \\
-\end{array}$ & $\begin{array}{r}34 \% \\
37-42 \% \\
\end{array}$ \\
\hline
\end{tabular}

Drug resistance was defined as any NNRTI mutation according to IAS mutation list 2019; Non-adherence was defined as mean VAS score <95\%; low level viremia defined as $50-1000 \mathrm{cps} / \mathrm{ml}$; - Not applicable

aNon-adherence among participants with viral suppression at 12 months; ${ }^{b}$ Non-adherence among participants with viral non-suppression at 12 months

* Stratified analysis due to significant interaction

${ }^{\beta}$ Cohort estimates are slightly larger than projected prevalences as they also take into account the effect of other predictor values included in the model 


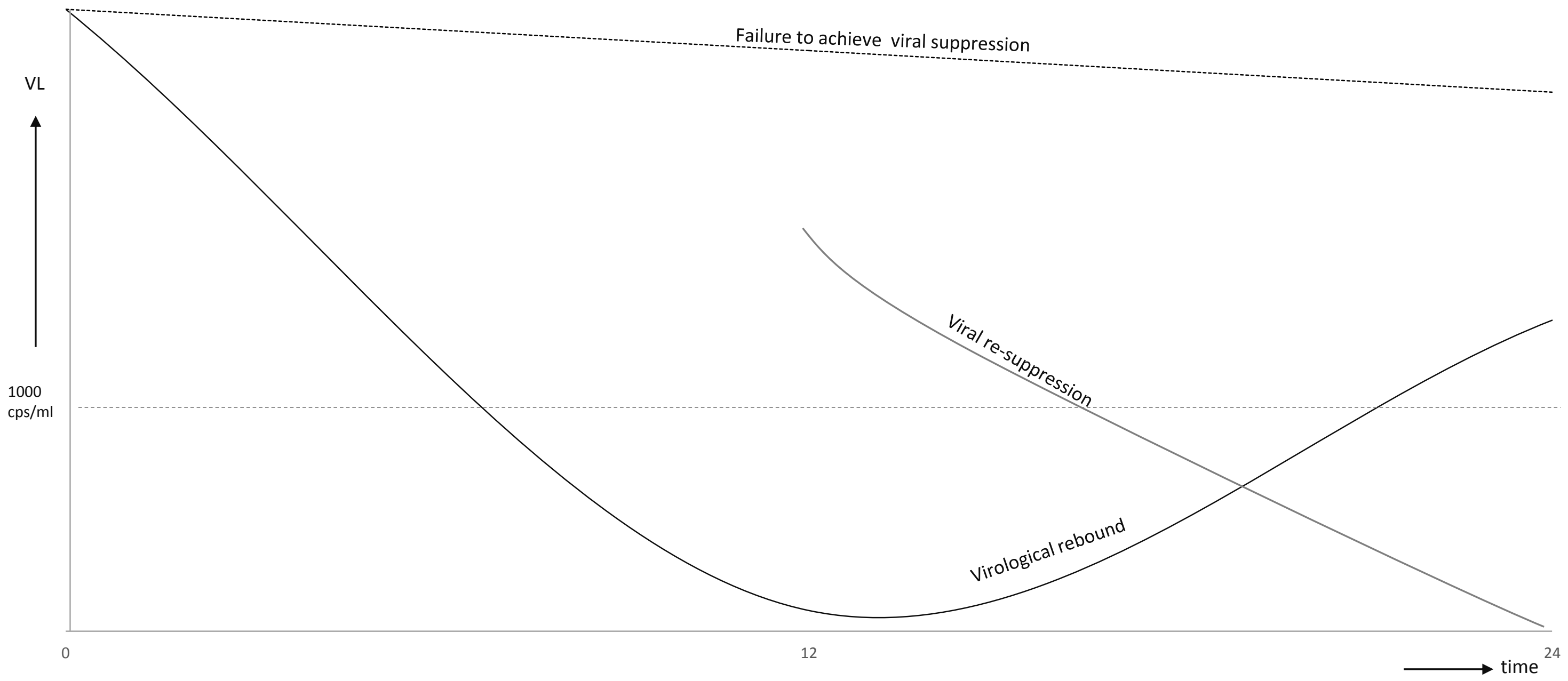

